\title{
Topological Phases Beyond the Hofstadter Butterfly
}

\author{
New theoretical work explores the phases that may arise when topology \\ intersects with the strong magnetic field effects presented by Hofstadter's \\ problem.
}

By Vidar Gudmundsson

M any materials exhibit topological phases.

In most cases, these robust states of matter arise from a complex band structure with spin-orbit interactions. But a simple band structure can also lead to topological effects when a strong magnetic field is applied to a two-dimensional lattice. This magnetically induced topology is captured by the Hofstadter butterfly-a fractal energy spectrum that maps out the competing influences of the field and the lattice on electrons. Although Hofstadter physics is well studied, little is known about the effect of a strong magnetic field applied to a material with an inherently complex band structure. In other

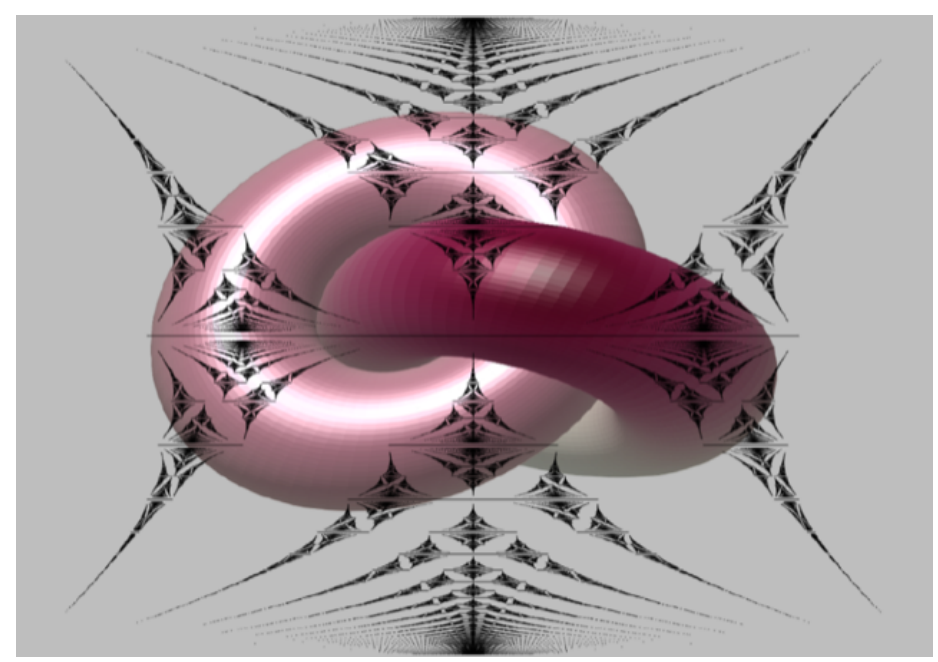

Figure 1: The Hofstadter butterfly (black) is shown here superimposed on an intertwined surface that is representative of topological phases in materials.

Credit: V. Gudmundsson words, what happens when the Hofstadter butterfly touches down on a topological material? Two separate theoretical groups have explored this question, both finding that novel phases of condensed matter may inhabit the overlapping region of Hofstadter and topology $[1,2]$. The results become more tantalizing in light of recent experimental work with moiré lattices-two-layered systems that have large effective lattice constants. These materials have the potential to bring some of the predicted Hofstadter topology to life.

The Hofstadter butterfly is the emblem of a long-standing problem concerning the single-electron states in a periodic electric potential and a transverse, constant magnetic field. The initial interest-going back 65 years-concerned metals in a uniform magnetic field, where it becomes difficult to construct the so-called Bloch wave functions that describe the behavior of electrons in a crystal lattice [3]. Subsequent work showed that this situation leads to the splitting of the quantized magnetic orbits (Landau levels) into subbands and the formation of semiclassical circular paths whose magnetic-field-dependent cyclotron radii extend beyond the lattice constant [4]. Further developments using a tight-binding model-an approach where the electrons live on a lattice with hopping probabilities to the neighboring sites-showed that the subbands depend on the magnetic flux passing through a lattice unit cell [5].

In 1976, Douglas Hofstadter revealed how the splitting of bands continues indefinitely as one varies the magnetic field, leading to bands within bands within bands. Plotted on a graph of electron energy versus magnetic field, the spectrum exhibits a butterfly-wing pattern (Fig. 1). This fractal structure is the result of the incommensurability of the two underlying length scales: 
the lattice constant and the cyclotron radius [6]. In simplified terms, gaps form in the band structure when electrons become "frustrated" trying to fit magnetic motion within the lattice spacings. To observe this frustration in a normal crystal, whose lattice constant is of order $0.1 \mathrm{~nm}$, would require an impractically large magnetic field of $1000 \mathrm{~T}$ or more. But in the 2000s, experimentalists were able to observe some of the coarse structures of the Hofstadter butterfly with fields of 0.4-12 T in AlGaAs-GaAs heterostructures, which can be imprinted with "lattices" having $100 \mathrm{~nm}$ spacing $[7,8]$.

Topological materials share some of their history with that of the Hofstadter butterfly. The discovery of the quantum Hall effect (QHE) in 1980 was a major breakthrough in the study of topological phases. Like the butterfly, this effect arises in a two-dimensional material exposed to a magnetic field. However, QHE-like edge states do not require an external magnetic field. Indeed, complex spin-orbit interactions can produce these conducting states on the surface of topological insulators in the absence of any external magnetic field. The study of topology has become a fruitful approach in condensed-matter physics, as evidenced by the 2016 Nobel Prize in Physics (see Focus: Nobel Prize-Topological Phases of Matter).

Opening a new chapter in this exciting field of topological materials, two theoretical groups-the first consisting of Andrei Bernevig from Princeton University and colleagues, the second made up of Jian Wang and Luiz Santos from Emory University in Atlanta-report on the emergence of a multitude of topological transitions in two-dimensional topological electron systems placed in an external magnetic field.

In their work, Bernevig and colleagues consider a variety of topological materials and how they might respond to a sufficiently large magnetic field [1]. The materials have a nonzero Chern number, which is a topological invariant that describes different classes of topological states. The group uses a tight-binding model for single-electron states to compute the topological phases that emerge in the Hofstadter spectrum for different symmetry conditions. Among the many phases that they predict, the researchers identify a 3D Higher Order Topological Insulating (HOTI) phase, in which the topologically protected current-carrying states are not along the edges (as in a normal topological insulator) but at the corners where edges meet.

Bernevig's team emphasize that their results are expected to be experimentally verifiable using moiré lattices, which are large-scale interference patterns formed by slightly rotating two identical overlaid patterns or lattices (see Trend: Bilayer Graphene's Wicked, Twisted Road). Like superlattices in AlGaAs-GaAs heterostructures, these patterns have large unit cells at small twist angles, thus relaxing the requirement of a strong magnetic field to produce Hofstadter splitting effects. Importantly, the researchers note two (as-yet-unpublished) experimental results with moiré lattices. The first reports signatures in twisted bilayer graphene of "fragile" Hofstadter topology, in which the traditional conducting edge states are missing [9]. The second looks at twisted double bilayer graphene (consisting of a bilayer graphene sheet laid over an identical bilayer) and identifies magnetic-flux-induced gap closings that are indicative of a Hofstadter semimetal defined by a topological variant in the bulk called the valley Chern number [10].

Wang and Santos take a different approach to Hofstadter topology [2]. They start with materials that exhibit Hofstadter effects-specifically Hofstadter-Chern insulators-and then explore the emergence of new topological phase transitions as the lattice parameters are tuned. An example of a Hofstadter-Chern insulator is a graphene layer on a boron-nitride substrate aligned to form a moiré pattern. The researchers assume the moiré pattern has a honeycomb structure (like graphene), and they employ an effective tight-binding description of the states. They find several topological phase transitions that arise from an interplay between Chern bands and van Hove singularities (non-smooth points in the density of states).

These fascinating developments have to be put into the context of related discoveries tied to the flat electronic bands that arise in moiré lattices [11]. For example, recent work has identified a wide range of phases-superconductivity, magnetism, and so on-in twisted bilayer graphene that researchers are busy deciphering (see Viewpoint: Geometry Rescues Superconductivity in Twisted Graphene). The electron dynamics in these new materials are still not well understood. For instance, it is not known at what point single-electron descriptions, like the tight-binding model, will need to be 
replaced with many-body descriptions. But it is exciting to follow where this close development in experiments and theory is taking the field of condensed-matter physics.

Vidar Gudmundsson: Science Institute, University of Iceland, Reykjavik, Iceland

\section{REFERENCES}

1. J. Herzog-Arbeitman et al., "Hofstadter topology: Noncrystalline topological materials at high flux," Phys. Rev. Lett. 125, 236804 (2020).

2. J. Wang and L. H. Santos, "Classification of topological phase transitions and van Hove singularity steering mechanism in graphene superlattices," Phys. Rev. Lett. 125, 236805 (2020).

3. P. G. Harper, "Single band motion of conduction electrons in a uniform magnetic field," Proc. Phys. Soc. London A 68, 874 (1955).

4. M. Y. Azbel', "Energy spectrum of a conduction electron in a magnetic field," Zh. Eksp. Teor. Fiz. 19, 929 (1964), [Sov. Phys. JETP 19, 634 (1964)].
5. D. Langbein, "The tight-binding and the nearly-free-electron approach to lattice electrons in external magnetic fields," Phys. Rev. 180, 633 (1969).

6. D. R. Hofstadter, "Energy levels and wave functions of Bloch electrons in rational and irrational magnetic fields," Phys. Rev. B 14, 2239 (1976).

7. C. Albrecht et al., "Evidence of Hofstadter's fractal energy spectrum in the quantized hall conductance," Phys. Rev. Lett. 86,147 (2001).

8. S. Melinte et al., "Laterally modulated 2D electron system in the extreme quantum limit," Phys. Rev. Lett. 92, 036802 (2004).

9. X. Lu et al., "Fingerprints of fragile topology in the Hofstadter spectrum of twisted bilayer graphene close to the second magic angle," arXiv:2006.13963.

10. G. W. Burg et al., "Evidence of emergent symmetry and valley Chern number in twisted double-bilayer graphene," arXiv:2006.14000.

11. L. Balents et al., "Superconductivity and strong correlations in moiré flat bands," Nat. Phys. 16, 725 (2020). 